\title{
Determination of Very Small Changes in Rate Over Intervals of Several Days in Mechanical Timepieces
}

\author{
By Horace A. Bowman
}

\begin{abstract}
A device for automatically plotting the isochronism of a clock or watch is described. It was developed to assist in precision tests on marine chronometers and watches, but it may be altered without difficulty to plot the curves for any timepiece. Its accuracy is 0.01 second, which is sufficient for ordinary timepieces, but by a few simple changes this accuracy may be increased. Some of the curves plotted by this instrument are shown. The sensitivity of this instrument, as well as its graphic scale, may be varied depending upon the quality of the timepiece being tested.
\end{abstract}

\section{Introduction}

The balance assembly of a watch is a mechanical oscillator, and like most oscillators, its resonant frequency changes with variations in the amplitude of oscillations. The amount of frequency variation that the balance assembly experiences as the watch runs down is known as isochronism. A curve in which the integrated time error of this oscillator is plotted as a function of the interval from winding is called the isochronism curve, or dial error, curve. As long ago as 1861 Phillips $^{1}$ stated the geometric conditions for the formation of a hairspring that would show no change in frequency of oscillation when the amplitude of oscillation is changed. These conditions are:

1. that the center of gravity of the terminal must fall on the radius that completes the first quadrant of the spring through which the curve passes, and

2 . that it must be distant from the center of the spring by an amount that equals $R^{2} / L$ : where $R$ is the connecting radius and $L$ the length of the theoretical terminal.

These conditions, while quite simple to state, are difficult to obtain in mass-production practice.

However, the theoretically isochronous hairspring terminal (sometimes called the "overcoil" because it is a coil of the hairspring that is carried above the main body of the spring from the outer edge and terminates on a nonoscillating part of the watch) is quite closely approached in the very fine watches manufactured today. The isochronous error is also reduced by increasing the power delivered by the mainspring, so that there is little change in the amplitude of oscillation of the balance wheel in the 24 hours that the watch is designed to keep good time. As a result, the isochronism error frequently drifts less than two or three hundredths of a second per hour. Plotting a curve by observing the watch once or twice per hour with the assistance of a chronograph and a standard of time will not show up these tiny drifts of rate. As timepieces improve, methods of testing must improve in order that the test may continue, to have meaning.

The Bureau's Time Section has developed an instrument that observes the watch every 0.4 second

1 M. Phillips, Memoire sur le spiral reglant des chronometers et des montres (1861). and plots the integrated time error to an accuracy of $1 / 100$ of a second.

\section{Design of the Instrument}

This instrument is essentially a relay-type servo system, which, by means of advancing or retarding the phase of a very accurate quartz-crystal-controlled frequency, keeps this standard frequency in phase with the frequency of the balance assembly of the watch being tested. The phase and frequency of the oscillator itself are unchanged by the action of this system. By plotting the amount of phase shift that is thus introduced into the standard frequency and choosing graphic coordinates referring to time rather than degrees of phase shift, a plot of the dial error is generated.

The standard frequency, accurate to 1 part in $50,000,000^{2}$ at a magnitude of $60 \mathrm{c} / \mathrm{s}$ and at low impedance, is passed through a continuous phase shifter $^{3}$ constructed from a Bendix two-phase selfsynchronous motor. (See fig. 1.) Clockwise rotation of the shaft of the motor results in advancing the phase of the output frequency, and counterclockwise rotation causes a retardation of the phase. One complete rotation of the shaft introduces a phase shift of $360^{\circ}$. This output frequency is passed through an amplifier and used to drive a 150-rpm synchronous motor. A galvanometer mirror is attached to the shaft of this motor. This mirror, rotating in a beam of light, causes light to fall alternately upon two vacuum photocells. The optical system is so arranged (see fig. 2) that the interval between the illumination of photocell $V_{1}$ and the illumination of photocell $V_{2}$ is 0.3 second, and the interval between the illumination of $V_{2}$ and the illumination of $V_{1}$ is 0.1 second.

The pulse formed by $V_{1}$ is passed through a triode connected as a cathode follower, and used to trigger a 2050 Thyratron, $V_{3}$. The audible tick of the watch is amplified and used to trigger a second Thyratron, $V_{4}$. The coils of two double-throw relays are inserted in the plate circuits of both $V_{3}$ and $V_{4}$, and the two relay coils terminate on one side of a condenser inserted in the $B+$ lead (see fig. 3 ).

2 J. M. Shaull, Adjustment of high-precision frequency and time standards, Proc. IRE 38, 6 (1950).

3 Invented by M. G. Pawley, Electronies and Ordnance Division, National Bureau of Standards. 


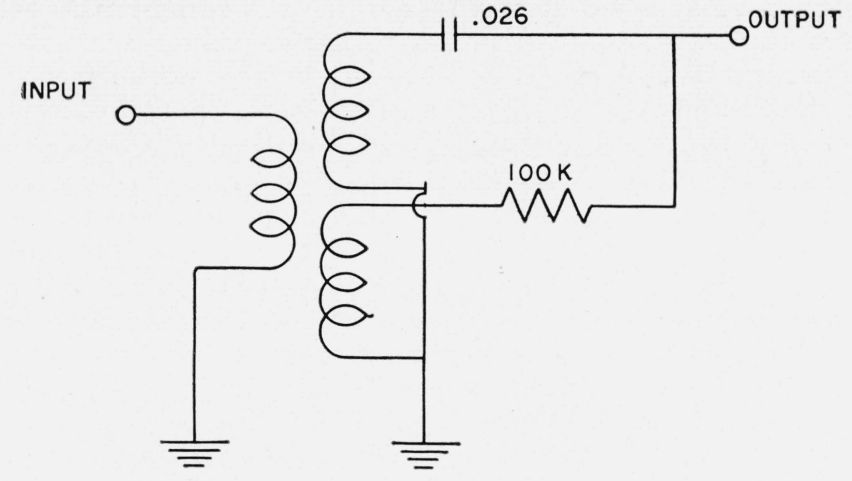

FIgURE 1. Phase shift circuit used with the isochronism recorder.

The condenser is chosen so that its capacitative reactance will equal the resistance at the input frequency. The values illustrated are for 60-cycle operation.

When a pulse from $V_{1}$ ionizes $V_{3}$, relay 1 closes (it is normally open) and remains closed as long as $V_{3}$ conduits. However, when the lower plate of $C_{1}$ is saturated with electrons, there being no other electron path available, $V_{3}$ deionizes and relay 1 opens. Now a pulse on the control grid of $V_{4}$ will not cause $V_{4}$ to ionize because a completely charged $C_{1}$ blocks the electron path. It is obvious that had the pulse on $V_{4}$ arrived before the pulse on $V_{3}, V_{4}$ would have ionized, closing relay 2 , and $V_{3}$ would have remained deionized. In other words, $V_{3}$ and $V_{4}$ act as a pulse selection circuit, closing either relay 1 or relay 2 depending on whether the pulse from the watch tick arrived before or after the pulse from the motormirror driven by the standard frequency (watch fast or watch slow).

With $C_{1}$ charged, additional pulses on $V_{3}$ and $V_{4}$ will cause no action; hence it becomes necessary to insert a mechanism to bleed the electrons from the lower plate of $C_{1}$. This is done by $V_{5}$, a 2050 Thyratron, which is triggered by the pulse from $V_{2}$ through a cathode follower. Relay 3 , with its points in series with its coil, is inserted in the plate circuit of $V_{5}$. This relay is normally closed and opens only

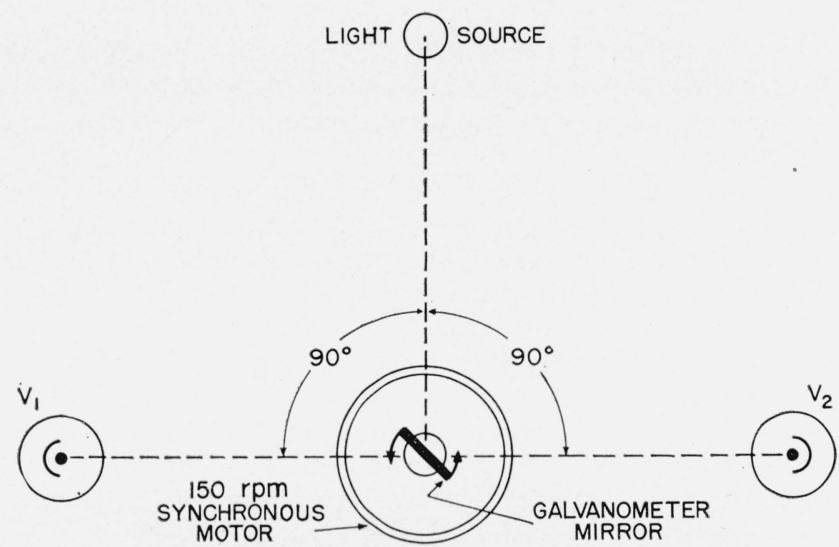

FIGURE 2. Diagramatic outline of the optical system used for the alternate illumination of $V_{1}$ and $V_{2}$.

At a motor-mirror speed of $150 \mathrm{rpm}$, the interval between the illumination of $V_{1}$ and $V_{2}$ is 0.3 second, while the interval from the illumination of $V_{2}$ to the illumination of $V_{1}$ is 0.1 second. when $V_{5}$ conducts. Its armature is heavily weighted to increase armature inertia. Hence when $V_{5}$ becomes ionized there is a sluggishness in the opening of relay 3. This permits the electrons on the lower plate of $C_{1}$ to escape completely to $+B$ through $V_{5}$ before the relay opens and destroys the electron path to $+B$. Likewise, the sluggishness of the armature of relay 3 permits $V_{5}$ to become completely deionized before it again closes the path to $+B$. This protects $V_{4}$ and $V_{5}$ from becoming permanently ionized by a burst of noise on $V_{4}$ at the same instant that $V_{5}$ is ionized.

Remembering that the pulse from $V_{2}$ is 0.3 second later than that from $V_{1}$, and realizing that only 0.2 second elapses, between the "tick" and the "tock" of a watch, if the tick of the watch triggered $V_{4}$, the tock, arriving 0.2 second later, would cause no action because $C_{1}$ would be still charged. In other words,

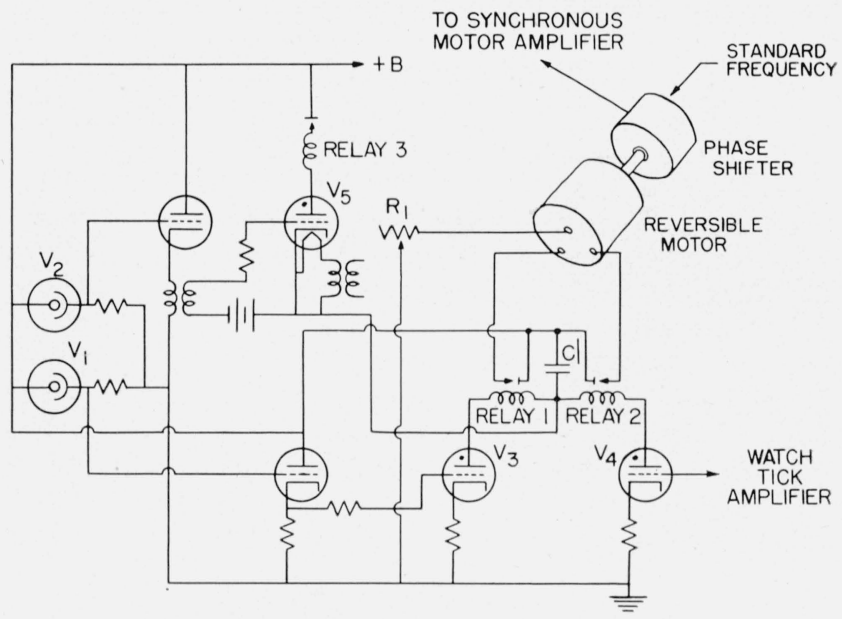

Figure 3. Simplified diagram of the isochronism recorder.

Due to cathode-filament potential separation limitations a separate bias and 1 and 2 and the reversible motor are drawn in simplified form. The true connec1 and 2 and the reversible motor are drawn in simplified form. The true connec-
tions are shown in figure 5. The input to the phase shifter is the standard frequency, and the output goes to the 150-rpm synchronous motor shown in figure 2.

this instrument will accept for comparison only the ticks or only the tocks. This condition was chosen because even watches in the best of condition have an interval from the tick to the tock different from the interval from the tock to the tick. Either the ticks or the tocks are suitable for comparison purposes, but if they were both accepted the instrument would register alternately "fast" and "slow", a condition that obviously does not exist within the watch.

A small, reversible motor is so connected that when relay 1 closes it rotates in a clockwise direction, and when relay 2 closes it rotates in a counterclockwise direction; thus a slow watch causes the motor to run clockwise, and vice versa.

The servo loop is completed by a mechanical coupling between the armature of the reversible motor and the armature of the phase shifter. Thus, the motor introduces motion into the phase shifter in such a manner that the order of pulses received by 

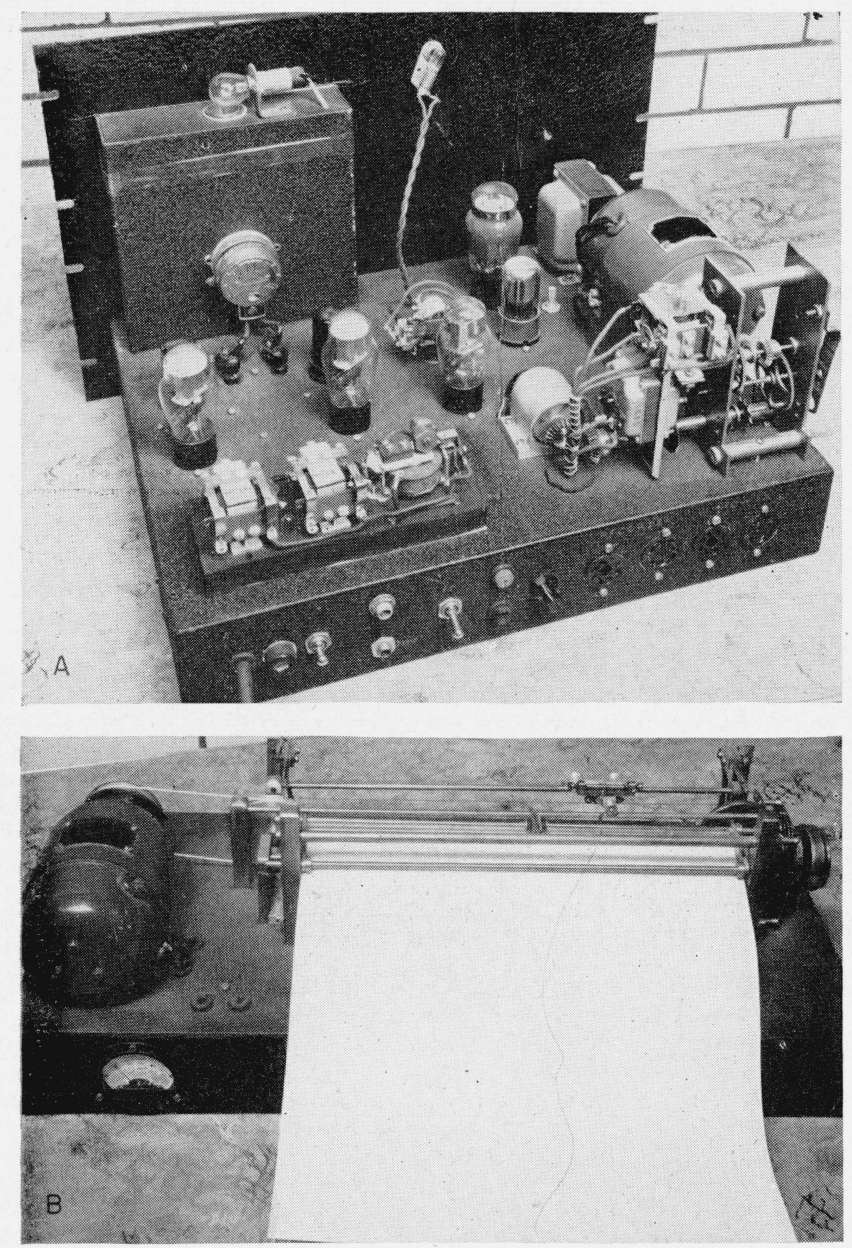

FiguRe 4. Isochronism recorder.

$A$, Relays 1,2, and 3 are at the front of the chassis and mounted on sponge rubber to minimize noise. The gear train performs two functions: first, it limits the amount of motion transmitted from the reversible motor to the phase shifter; and second, it controls the motion transmitted to the recording section via a selfsynchronous motor.

$B$, Recording section of the isochronism recorder. The self-synchronous motor at the left is controlled by a similar one just behind the gear train in $A$.

the comparison circuit, $V_{3}-V_{4}$, tends to be reversed. A gear train (see fig. 4, $A$ ) reduces the amount of motion transmitted to the phase shifter by the motor.

The motion of the phase shifter is transmitted via a self-synchronous motor system to a remote recorder (see fig. 4, B) where one complete revolution of thep hase shifter is plotted as $1 / 60$ of a second. Rheostat $R_{1}$ (fig. 3 ) varies the voltage dropping across the reversible motor and thereby acts as a sensitivity control, governing the amount of motion performed by the motor for each impulse received.

Relays 1 and 2 are connected as indicated in figure 5. This type of connection is used to prevent both a clockwise and a counterclockwise impulse being applied to the motor at the same instant. Condenser $C_{1}$ (fig. 3) is chosen so that it will store enough energy at the plate voltage used to keep relays 1 and 2 closed 0.005 second. This means that if the pulse arrival from the photocell differs by less than 0.005 second from the pulse arrival from the watch tick amplifier, both relays will partially act because there will be enough positive charge on the lower plate of $C_{1}$ to permit both $V_{3}$ and $V_{4}$ to ionize. This is the factor that limits the sensitivity of the instrument, as the comparator cannot distinguish between pulses separated by less than 0.005 second. If greater sensitivity is required, a smaller condenser should be used in place of $C_{1}$. The value of $0.2 \mu \mathrm{f}$ (the actual value of $C_{1}$ in this instrument) is not a limiting value. In the development of this instrument values as low as $0.05 \mu$ f were used, with correspondingly smaller relays and a more sensitive motor, with quite satisfactory results; however, it was felt that the larger, more rugged motor and relays were desirable for a constantly working laboratory instrument.

Considering the other errors in the instrument, such as the lag in the self-synchronous-motor system that transmits the information to the recorder, the stylus friction in the recorder, et cetera, the instrument has an accuracy of \pm 0.01 second at any time. As stated previously, this accuracy could be consider-

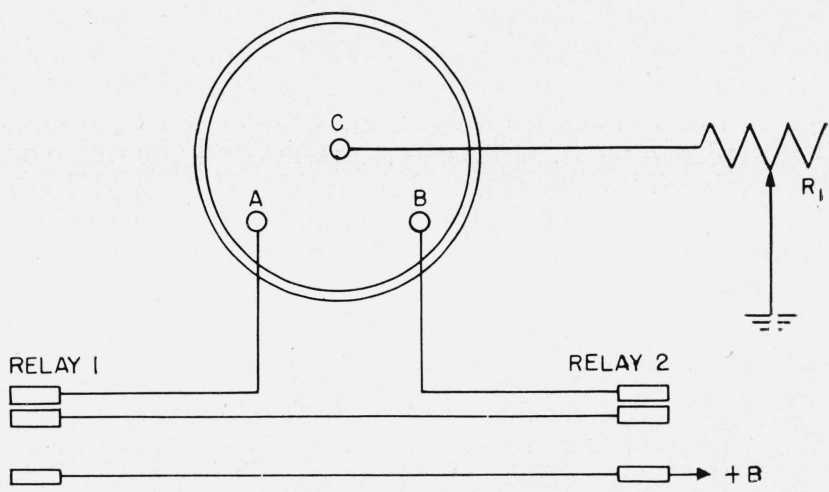

Figure 5. Motor-control relay connections.

Details of the connections between the reversible motor and the double-throw relays 1 and 2, which control its direction of motion. When a pulse is applied between $A$ and $C$ on the motor, the armat or between $A$ and $C$ on the motor, the armature rotates counterclock wise while the pulse is being applied. When a pulse is applied between $B$ and $C$, the motion is clockwise. By making the connections as illustrated, the motor is protected against having both a clockwise and counterclockwise impulse applied at the ame instant. The relay points are shown in their normal position. $R_{1}$ is the sensitivity control that governs the amount of motion executed by the motor for any one impulse.

ably increased by certain mechanical changes, but since watches do not repeat observations with great precision, no effort is contemplated to increase this value.

Upon examination of figure 3, several simplifying devices become apparent. The elimination of relays 1 and 2 can be accomplished and the pulse actuating them can be applied directly to the reversible motor, the only change necessitated thereby being the enlargement of condenser $C_{1}$. The photoelectric system performs two functions: First, it eliminates alternate ticks, and second, it controls the drain of electrons from the lower plate of $C_{1}$. The separation of ticks from tocks could be accomplished by a flip-flop and the discharge of $C_{1}$ by a bleeder resistor. The more complicated arrangement was used because this instrument will be called upon in future problems to control electric motors too large for convenient Thyratron drive. 


\section{Use of the Instrument}

This instrument is used in plotting the isochronism curves that accompany class A watch tests performed by the Bureau. The 150-rpm motor may be changed to a 120 -rpm motor, thereby making it possible to plot the isochronism curve of a marine chronometer. Provisions have been made on the instrument to make this change quickly and conveniently. For that matter, it is possible to make a plot of the isochronism of any timepiece as long as the revolutions per minute of the motor just equal the nominal value of the balance frequency (remembering that in most watches the "beat" of a watch equals twice the balance frequency).

To use the instrument, a watch is wound and placed on the vibration pick-up provided. The the balance wheel. This noise, the first, although not a loud noise, is caused by the roller jewel (attached to the balance wheel) striking the fork slot. The position of the loudest noise on the time axis becomes quite erratic in the minutes preceding the complete run-down of the mainspring. In order to assure sufficient amplification to receive the first noise, a pentode and a triode are connected together in a two-stage microphone amplifier.

Two neon indicators show when the instrument has accomplished synchronization. One of them is connected across relay 1 , with sufficient series resistance to prevent burnout, and the other across relay 2 . When both lights flash, the instrument is holding the standard frequency in phase with the watch.

This isochronism plotter may be used as an ordi-

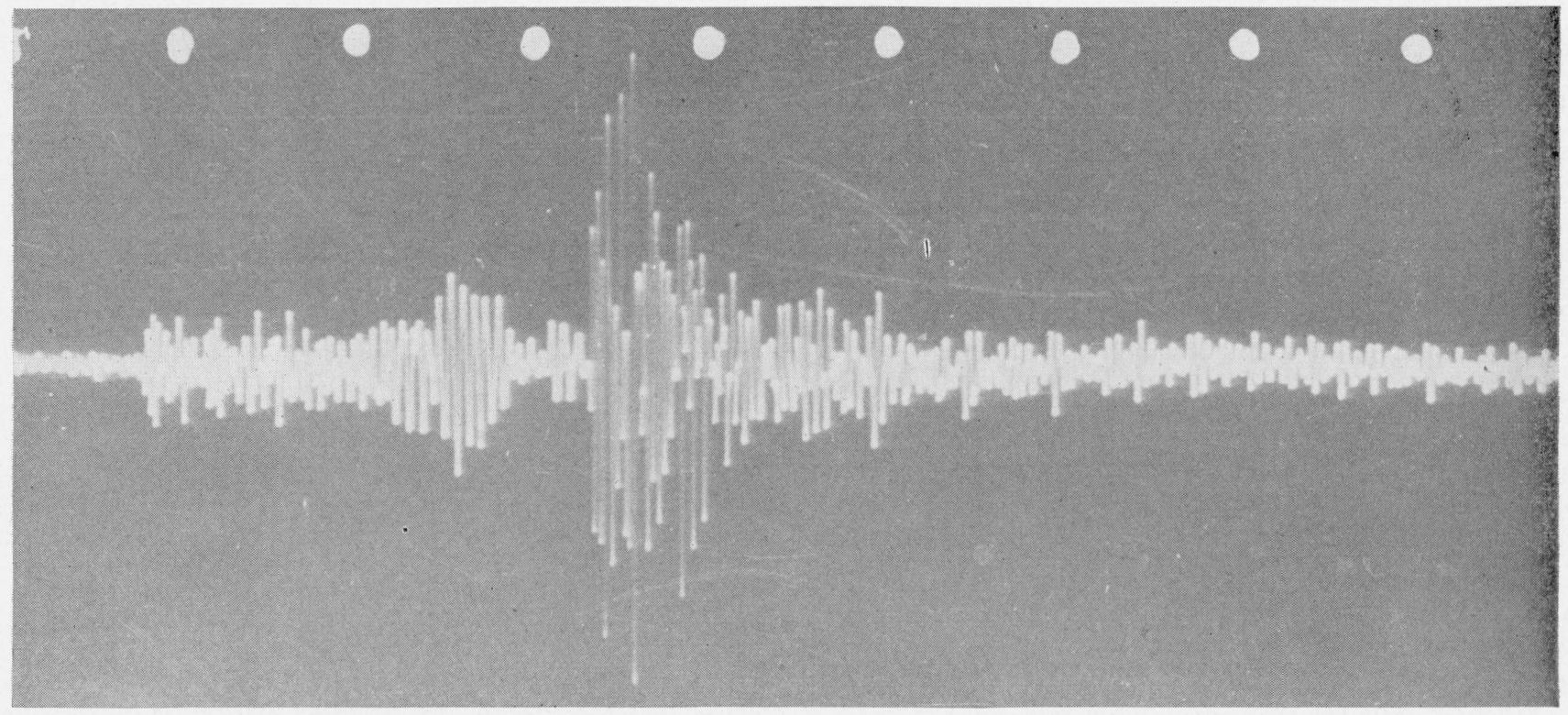

Figure 6. Oscillogram of a watch tick

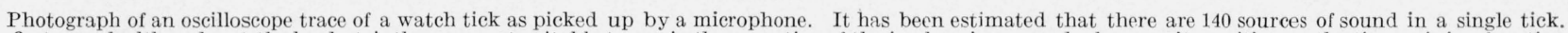

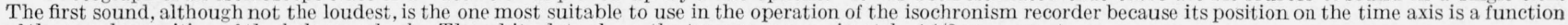
of the angular position of the balance wheel. The white dots above the trace are approximately $41 / 2 \mathrm{msec}$ apart.

instrument will take about one minute in "hunting" for the watch tick or tock. The noise that occurs during the first coincidence in the comparator circuit is thereafter held in synchronization whether it is the tick or the tock. Thereafter the servo loop keeps the standard frequency in phase with this particular noise.

In each tick or tock there are several important noises to consider (see fig. 6) that are caused by the action of the balance assembly.

The loudest noise is the dropping of the escape wheel tooth onto the locking face of the pallet stone. This noise depends upon the mainspring force for both its amplitude and position on the time axis, so it is not a particularly good noise on which to operate.

Since we are primarily concerned with the frequency of the balance wheel, it is best to use a noise that always occurs at the same angular position of nary watch rate recorder to give the instantaneous rate of the watch if desired. Inasmuch as the plot generated by the recorder is the integrated time error, the slope of this curve is the instantaneous rate. In this respect it gives a clearer picture of the instantaneous rate than a regular rate recorder, because in watches in which the train friction varies widely, the operator may draw a smooth curve through the true irregular curve and determine a "better" instantaneous rate.

Figure 7 illustrates four isochronism curves plotted by this instrument. They are for the same watch in the same position (dial up). The irregularities in these curves of less than 0.1 second would have escaped notice had these curves been plotted by a chronograph, even with the assistance of a highprecision standard of time. On a 24-hour basis this watch was running at a rate constant to $1.80 \mathrm{sec} / \mathrm{day}$. 
The adjustment of a watch is intended to eliminate the differences in the rate when it is operated in various positions. Figure 8 shows the actual differences in rate of a newly cleaned and overhauled 19-jewel, size 12 , pocket watch. If the watch had been in "perfect" adjustment and contained a "perfectly" isochronous hairspring, the six curves would have been superimposed one upon the other. The variations are caused by frictional changes and gravitational effects that deform the hairspring from its truly isochronal configuration. It might be pointed out here that isochronism tests are usually performed with the watch in either the dial up or dial down position.

In addition to the routine testing for which this instrument was designed, it is being used in researches currently being conducted upon the effects of friction in the train, balance lubrication, and variations in amplitude. It plots curves that are sufficiently accurate to permit differentiation and interpretation. It has eliminated the necessity of a long vigil with a watch, reading it at regular intervals and recording the observation on a chronograph in order to obtain the information necessary to plot an isochronism

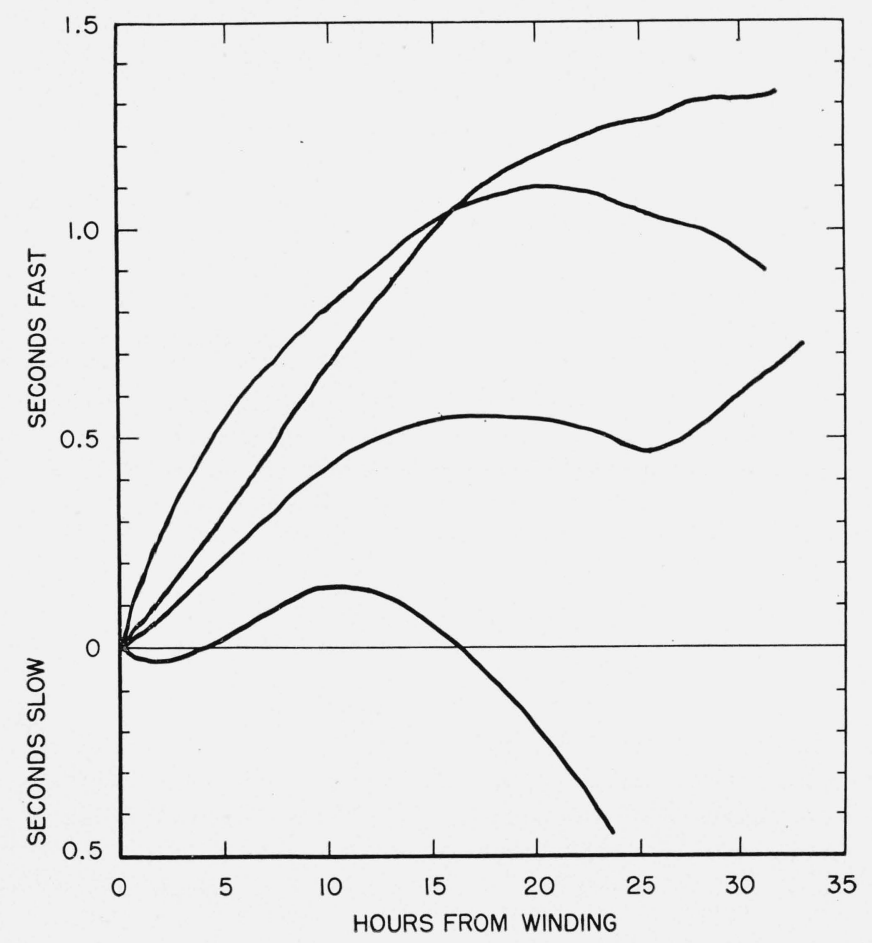

FIGURE 7. Four successive isochronism curves on the same watch.

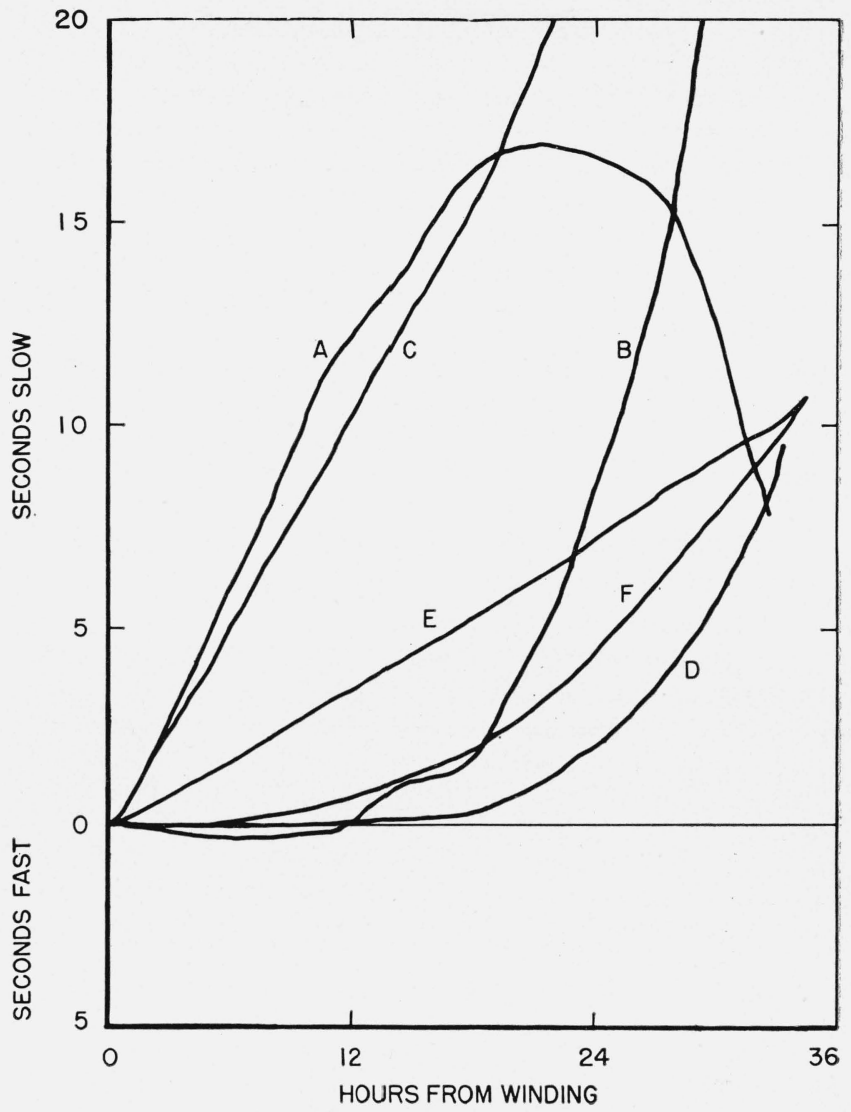

FiguRe 8. "Run-down" curves plotted for a newly cleaned and overhauled size 12, 19-jewel pocket watch.

This watch is poorly adjusted since placing it in various positions for test results in widely diverging isochronism curves. Ordinarily the isochronism curve is drawn for the "dial up" and "dial down" positions only.

$A$, Pendant right; $B$, pendant left; $C$, pendant down; $D$, pendant up; $E$, dial up; $F$, dial down.

curve. Under the previously used procedure the factors of human fatigue limited the accuracy of any observation to \pm 0.2 second, and since the watch was seldom observed more frequently than once each hour, the curve finally arrived at was comparatively crude. The benefit of an observation every 0.4 second, accurate to \pm 0.01 second, permits the plotting of a curve upon which the most exacting analysis may be conveniently performed.

The criticisms, comments, and assistance given by C. N. Challacombe, Research Physicist, Elgin National Watch Co., are gratefully acknowledged.

Washington, April 11, 1950. 